\title{
Análisis de grupo y evaluación financiera de unidades productivas de cebolla de bulbo (Allium cepa L.) en el Distrito de Riego del Alto Chicamocha (Boyacá-Colombia)
}

\section{Analysis of group and financial evaluation of productive units of bulb onion (Allium cepa $L$.) in the District of Irrigation of Alto Chicamocha (Boyacá-Colombia)}

\section{Análise de grupo e avaliação financeira da produção de unidades bulbo da cebola (Allium cepa L.) no Distrito de Irrigação Alta Chicamocha (Boyacá-Colômbia)}

\begin{abstract}
Nancy Cristina Sanabria Neira ${ }^{1}$, Gloria Acened Puentes Montañez² \& José Alejandro Cleves Leguízamo³
${ }^{1}$ Administradora de Empresas Agropecuarias, Especialista en Gerencia Financiera, Magister en Administración Económica y Financiera. ²Especialista en Proyectos de Desarrollo, Magister en Ciencias Agrarias. ${ }^{3}$ Ingeniero Agrónomo, MSc en Fitotecnia, estudiante Doctorado en Agroecología

1,2,3 Escuela de Administración de Empresas Agropecuarias. Universidad Tecnológica y Pedagógica. de Colombia UPTC.
\end{abstract}

1nancycristinasa@gmail.com, 29lorispuentes@hotmail.com, 33clevesalejadro@yahoo.com

\section{Resumen}

En Colombia el cultivo de cebolla de bulbo (Allium cepa L) es un importante generador de ingresos y mano de obra para pequeños cultivadores en los departamentos de Boyacá, Nariño y Cundinamarca. Su producción es de $320.000 \mathrm{t}_{\text {año-1}}{ }^{-1}$, de las cuales $185.000 \mathrm{t}(58 \%)$ son producidas por el departamento de Boyacá, convirtiéndose en el primer productor a nivel nacional. El Distrito de Riego del Alto Chicamocha (DRACH) produce entre 35.000 a $40.000 \mathrm{t}$, es decir, $12,5 \%$ del volumen nacional y $21,62 \%$ del departamental, este renglón productivo es el más relevante en el área de influencia del distrito y es la principal fuente de ingresos y empleos tanto directos como indirectos. Se usó la Metodología Análisis de Grupo para desarrollar el objetivo general de la investigación de determinar la rentabilidad en las diferentes unidades; se reco- lectó información en fuentes primarias tales como: relaciones sociales, estructuras de costos, aspectos de manejo técnico y administrativo, así como la productividad obtenida. Se encontraron tres grupos de rentabilidad de las diferentes unidades: cabeza, media y cola. Se concluye que los costos de producción de la cebolla de bulbo se clasifican en explícitos (mano de obra, insumos, servicios y otros) e implícitos (trabajo familiar, arrendamiento, depreciación y renta del capital). Se propone a los propietarios de las unidades productivas del grupo de la cola, optimización del riego, uso apropiado de agroquímicos y realización de prácticas culturales oportunas.

Palabras Clave: análisis de grupo, análisis financiero, Distrito de Riego del Alto Chicamocha (DRACH) 


\section{Abstract:}

In Colombia the cultivation of bulb onion (Allium cepa L) is a major generator of incomes and handwork for small growers in the departments of Boyacá, Nariño and Cundinamarca. Its production is $320,000 \mathrm{t}^{\text {year }}{ }^{-1}$, of which $185,000 \mathrm{t}$ (58 \%) are produced by the department of Boyacá, becoming the first producer at national level. The Irrigation District of the Alto Chicamocha (Drach) produces between 35,000 to 40,000 t, i.e. $12.5 \%$ of the national volume and $21.62 \%$ of the departmental one, this productive line is most relevant in the influence area of the district and it is the main source of income and employment both direct and indirect. It was used the methodology of group analysis to develop the general objective of this research to determine the profitability in the different units; information was collected on primary sources such as: social relations, cost structures, aspects of technical and administrative management, as well as the productivity gained. It was found three groups of profitability of the different units: head, medium and tail. It was concluded that the production costs of the onion bulb are classified in explicit (handwork, inputs, services, and others) and implicit (family work, lease, depreciation and capital income). It is proposed to the owners of the production units of the cola group, the optimization of irrigation, the appropriate use of agrochemicals and the implementation of appropriate cultural practices.

Key-words: group analysis, financial analysis, Irrigation District of the Alto Chicamocha (DRACH)

\section{Resumo:}

Na Colômbia, o cultivo do bulbo da cebola (Allium cepa $\mathrm{L}$ ) é uma importante fonte de renda e trabaIho para os pequenos agricultores nos departamentos de Boyacá, Cundinamarca e Nariño. Sua produção é de $320.000 \mathrm{t}$ ano-1, dos quais $185 \mathrm{mil}$ t $(58 \%)$ é produzido por Boyacá, tornando-se o primeiro produtor nacional. O Distrito de Irrigação de alta Chicamocha (DRACH) produz entre 35.000 a 40.000 t, ou seja, $12,5 \%$ do volume nacional e $21,62 \%$ do departamento, esta linha de produção é a mais relevante na área de influência do distrito sendo a principal fonte de renda e emprego, entre diretos e indiretos. Foi usada a metodologia de análise de grupo para desenvolver o objetivo geral da pesquisa de determinar a rentabilidade em unidades diferentes; Os dados foram coletados em fontes primárias, tais como as relações sociais, estruturas de custos, aspectos de manejo técnico e administrativo, assim como a produtividade obtida. Encontraram-se três grupos de rentabilidade das diferentes unidades: cabeça, media e cauda. Conclui-se que os custos de produção da cebola de bulbo são classificados em explícitos (mão de obra, materiais, serviços, etc.) e implícitos (mão de obra familiar, renda, depreciação e ganhos de capital). Se propõe aos proprietários de unidades produtivas do grupo da cauda otimização da irrigação, o uso adequado de agroquímicos e realização de práticas culturais adequadas.

Palavras-chave: análise de grupo, análise financeira, Distrito de Irrigação de Alta Chicamocha (DRACH)

\section{Introducción}

La rentabilidad está asociada a una concepción empresarial que genera crecimiento e innovación en las diferentes regiones del país y particularmente en el departamento de Boyacá, las unidades productivas no pueden sustraerse a los fenómenos económicos, sociales, ambientales, las políticas del gobierno, la opción tecnológica disponible y la competencia, que permitan lograr la sostenibi- lidad de las agroempresas. Es importante orientar la toma de decisiones para la generación y maximización de valor de las unidades productivas, buscando su posicionamiento sólido y creciente en el mercado, desarrollando productos y servicios que garanticen la generación de flujos monetarios constantes y crecientes, generando mayor rentabilidad para los propietarios (Alizaga, 2007). 
En la presente investigación, el objeto de estudio son las unidades productivas de cebolla de bulbo Allium cepa $L$ localizadas en el Distrito de Riego del Alto Chicamocha (DRACH) en la Provincia del Tundama, departamento de Boyacá. Para el análisis financiero, se evaluaron las principales variables involucradas en los procesos productivos (Tabla 1). Se parte de establecer la situación real de las unidades productivas, para lo cual se determinan las prácticas técnicas, económicas y administrativas asociadas a la rentabilidad, identificando las limitaciones y generando un sistema de información que posibilite la toma de decisiones a los actores involucrados de acuerdo a las tendencias o proyecciones del mercado (Pool, 2013).

\section{La gestión en el agronegocio de la cebolla}

El "sistema de producción agropecuaria" es una unidad compuesta por elementos que interactúan entre sí y con su entorno medioambiental y cultural (Martínez \& Alemán, 2004).

El enfoque de sistemas aplicado a la agricultura permite identificar restricciones y oportunidades estratégicas para el desarrollo rural y su poder subyace en la habilidad para integrar análisis multidisciplinarios de producción y su relación con recursos, tecnologías, mercados, servicios, políticas e instituciones en su entorno local, la nueva concepción de la gerencia desafía el problema de asegurar la posición competitiva de las unidades productivas, el conocimiento del mercado y la utilización óptima y racional de los factores productivos, por tanto, se requiere de la constante adaptación a ambientes cambiantes buscando alternativas de crecimiento (Mena, 2012).

La operación de las empresas o unidades productivas implica llevar a cabo una serie de actividades o funciones: mercadeo, producción, gestión del talento humano y finanzas, dependiendo del tamaño, definición y el modelo del negocio; pero siempre respondiendo a unos objetivos organizacionales preestablecidos que sirvan como guía para la toma de decisiones de gestión (Lerdón, 2010).

La gestión financiera se basa en decisiones que contribuyan al fortalecimiento del área de capital con el fin de alcanzar el objetivo financiero básico de maximizar el valor de las empresas, apuntando permanentemente hacia el logro de un crecimiento sostenido (crecer y permanecer) con base en la generación de ingresos y la eficiencia en el manejo de los recursos ofreciendo al gerente infinidad de opciones que obedezcan a una estrategia planificada a largo plazo, definida en función de los propietarios y en armonía con los objetivos asociados con los clientes, trabajadores y demás grupos de interés (García, 2009).

Las unidades empresariales deben aumentar su valor en mayor proporción a lo invertido, alcanzando rentabilidad y generando flujos de caja suficientes para su operación. A través de la planeación y el control financiero, la actualización tecnológica, la evaluación económica de actividades de inversión, la sincronización perfecta de los flujos monetarios, la integración de los recaudos y las disponibilidades iníciales de efectivo que permitan el cumplimiento oportuno de los compromisos de deuda, favoreciendo el mantenimiento de las buenas relaciones laborales, comerciales y financieras, propende por el uso eficiente de los recursos (Ortiz, 2005).

El componente financiero se abordó a partir de la fundamentación teórica de la gestión financiera, como es el registro de las operaciones empresariales, con especificidad en la contabilidad agrícola. El uso de la contabilidad en las empresas agrícolas permite obtener comprensión del resultado económico y tomar decisiones sobre su continuidad, también es importante para cumplir con los requisitos del pago de impuestos, planificar el mejoramiento de la infraestructura de la finca, tener conocimiento sobre la gestión empresarial y la rentabilidad del negocio así como en la solicitud y obtención de financiamiento, en busca de lograr 
mejores resultados (Ortiz, 2005). La integración de la información se logra a través de los Estados Financieros, estos relacionan cifras monetarias presentadas con un ordenamiento determinado según su propósito (Ortiz, 2006)

En las empresas agropecuarias el mayor porcentaje de sus costos están destinados a la producción, ya que ésta es la que va a determinar la utilidad bruta por unidad de tiempo. Para el fundamento tanto de los estados financieros como del cálculo y análisis de la rentabilidad del cultivo de cebolla de bulbo se requiere de los insumos principales para su elaboración como son los costos, ya que toda empresa necesita los recursos para adquirir tanto los insumos como los medios de producción (Ugalde \& Esquivel, 1986).

En general se denomina rentabilidad a la medida del rendimiento que en un determinado periodo de tiempo producen los capitales utilizados en un proceso productivo (Brian, 2010).

\section{Análisis de grupo}

Este método de gestión establecido en Francia por el profesor Chombart en 1965, ayuda a que el productor pueda elegir un sistema de producción que le permita obtener los mayores beneficios dependiendo del medio, la coyuntura y la posibilidad de éste para establecer adecuadas comparaciones y combinaciones rentables (Chombart, 2000).

El método de análisis de grupo, pertenece a los métodos analíticos, éste requiere la constitución de una zona homogénea, donde se analiza un grupo de explotaciones bajo ciertos criterios que varían de acuerdo a las características de la región, procurando que las unidades productivas sigan el mismo sistema de producción (García, 2009). De acuerdo a los resultados, las explotaciones se dividen en tres categorías: explotaciones de cabeza, promedio y cola. En la categoría cabeza se obtiene los mejores resultados económicos, por otra parte, las explotaciones clasificadas dentro de la categoría cola son las explotaciones en donde se encuentran deficiencias graves que limitan los resultados económicos. Finalmente se proponen soluciones de optimización.

\section{Aspectos agroecológicos de la cebolla de bulbo}

Hay variedades de cebollas específicas para las diferentes zonas, la selección de la variedad a sembrar depende fundamentalmente del fotoperiodo y este depende de la geoposición en el globo terráqueo, así se tienen zonas de día corto (10 o menos horas-sol por día), zonas de día intermedio (10-12 horas-sol por día) y zonas de día largo (hasta 16 horas-sol por día) (MADR, 2002).

Por el alto valor de las semillas, en Colombia el uso de semilleros es el método de propagación más común. En este proceso se acondicionan eras denominadas "camas de propagación" es un suelo mullido, rico en materia orgánica, en donde se dispone de condiciones adecuadas que estimula el proceso de germinación y posterior desarrollo radicular, el lugar cuenta con un sistema de riego localizado. Esta etapa es definitiva por que el mantenimiento define en gran medida el crecimiento y desarrollo del cultivar. Este periodo fenológico es muy importante ya que requiere un alto uso de mano de obra fundamentalmente de carácter familiar. Cuando la planta tiene una altura entre 20 a $25 \mathrm{~cm}$ se procede al trasplante al sitio definitivo (Álvarez, 2011)

\section{Antecedentes de los estudios económi- co-financieros en la cebolla de bulbo}

En Guatemala la Universidad de San Carlos (2002), realizó un estudio relacionado con la rentabilidad de la producción del cultivo de la cebolla de bulbo, encontrándose productores que no conocían la rentabilidad de sus producciones, situación que llevo a definir un estudio para establecer los principales aspectos financieros del cultivo durante los años 1999-2002, se efectuaron 
proyecciones y se evaluó financiera y económicamente el cultivo, precisando el rendimiento.

El trabajo de Cristianoy Sastre (1997), encontró un beneficio económico aplicando la técnica física de la radio inhibición que consiste en el control al rebrote de la cebolla en condiciones de almacenamiento, lo cual permite ofertar el producto en concordancia con los volúmenes ofertados y con el precio en los mercados.

En Colombia la Corporación Autónoma Regional del Tolima (CORTOLIMA) en el año 2006, estructuro el Plan de Ordenación y Manejo de la Cuenca Hidrográfica del rio Coello (POMCA), para este fin diseño un convenio interadministrativo con el concurso de Corporación de Investigación Agropecuaria (CORPOICA), el Servicio Nacional de Aprendizaje (SENA) y la Universidad del Tolima, en este estudio se elaboraron estudios de factibilidad en hortalizas, (cebolla de bulbo) a través de la preparación de planes de negocios, organización comunitaria, mejorando la infraestructura y el proceso de transferencia tecnológica.

La Sociedad Colombiana de Ciencias Hortícolas (SCCH) en el año 2009 organizó el III Congreso Colombiano de Horticultura y el I Simposio Internacional de Cebolla y Ajo en el Trópico con el propósito de compartir y dar a conocer las últimas investigaciones en el desarrollo del sector tanto a nivel nacional como internacional, en aspectos de costos y evaluación financiera, ensayos con diferentes fertilizantes, formas de almacenamiento, poscosecha, sistema de gestión de calidad para la cebolla.

En el departamento de Boyacá, se han realizado diferentes estudios relacionados con el cultivo de cebolla de bulbo como producto de importancia en el sector agropecuario, cabe mencionar entre estos los siguientes: Estudio de factibilidad para el establecimiento de un "packinghouse" para cebolla de bulbo (Allium Cepa L.) en el Distrito De Riego Del Alto Chicamocha (Martínez, 2009), con el objeto de identificar la posibilidad de crear un centro de acopio que permita realizar todos los procesos de poscosecha de la cebolla producida en el DRACH; Manual técnico del cultivo de la cebolla puerro (Allium porrum) bajo el uso de buenas prácticas agrícolas (BPA) en el distrito de riego del alto Chicamocha DRACH (Fonseca et al., 2008), como alternativa de diversificación y obtención de mejores productos en el Distrito; competitividad del sistema agroalimentario de la cebolla de bulbo con enfoque de cadena productiva en el Distrito de Riego Del Alto Chicamocha.

Melo (2008), efectuó un análisis del cultivo como parte de la cadena agroalimentaria identificando los aspectos que generan competitividad al producto. Puentes \& Cleves, (2010) realizaron investigaciones sobre el proceso de la poscosecha de cebolla de bulbo (Allium cepa L.) en el Distrito de Riego del Alto Chicamocha y efectuaron un análisis financiero del sistema de producción de cebolla de bulbo (Allium cepa L.) en el municipio de Corrales (Boyacá), identificando los costos de producción, ingresos, utilidades y rentabilidad del cultivo.

Fonseca et al. (2008); Fonseca \& Cleves, (2010), asociaron los sistemas de gestión de la calidad como un elemento para aumentar la competitividad en el cultivo de cebolla de puerro (Allium porrum L.) y en la producción de ajo y cebolla en Colombia, como un elemento diferenciador en el mercado nacional e internacional.

\section{Metodología}

Las etapas que se desarrollaron en el proyecto fueron las siguientes:

- Identificación del sistema de producción.

- Caracterización del sistema de producción de cebolla de bulbo.

- Conformación de grupos con características homogéneas (cabeza, media y cola) y análisis de Gestión. 


\section{Población y muestra}

Se aplicó una muestra probabilística a las unidades productivas, según censo de los productores adscritos al DRACH a abril de 2010.

Muestra: Para determinar la variable $n=42$ unidades productivas de cebolla de bulbo, se analizaron los siguientes aspectos teniendo en cuenta la selección de un grupo homogéneo de agroempresas:

- Estar ubicadas en el DRACH y pertenecer a una de las unidades de riego.

- Corresponder a los diferentes grupos identificados (grandes, medianas y pequeñas unidades productivas).
- Exteriorizar su aceptación para participar en el estudio, al contestar la encuesta.

Cálculo de la muestra

Para la determinación de la muestra, se utilizó la fórmula estadística para poblaciones finitas, con un nivel de confianza del $95 \%$ y un margen de error del 0,05 , con probabilidad de éxito del $50 \%$; así se encontró que el número de unidades a trabajar fue de $n=42$.

Las variables permiten medir los objetivos y deben estar contenidas en el instrumento de toma de información, para este caso se establecieron las indicadas en la Tabla 1.

Tabla 1. Sistema de Variables, establecido para las unidades productivas del DRACH

\section{Variables dependientes}

Productividad:

Rentabilidad:

Mercadeo, comercialización;

Administración:

Gerencia del Valor:

\section{Variables independientes}

Tecnología (riego, labores culturales control fitosanitario, fertilización)

Variedades empleadas, Sistema de producción, Características del mercado, Fenómenos naturales y desastres, Deterioro del Suelo

Rentabilidad, Costo de Producción, Costo de Capital, Ingresos Utilidad Operacional y Neta, recuperación del suelo

Canales de comercialización, Precios, Oferta y demanda Organización para la comercialización

Registros, Funciones, Flujogramas, Proceso administrativo

Rentabilidad, TIR, VPV, Costo de Capital, Eva, Flujo de Caja Libre.

\section{Resultados y discusión}

Los datos corresponden a un estudio transversal que se llevó a cabo en las unidades productoras de cebolla de bulbo, con una población de 54 unidades (Censo del 2010). Para obtener una muestra representativa se aplicó un diseño muestral probabilístico aleatorio de tipo descriptivo, se calculó un tamaño de muestra de 42 unidades, con un margen de error de $5 \%$ y una confiabilidad de 95\%. Se aplicó como instrumento de medición una encuesta con 25 preguntas relacionadas con el sistema de variables indicadas en la Tabla 1.

Se recogió información relativa a variables técnicas y financieras medidas a través de preguntas 
abiertas cerradas. Los datos fueron procesados en el paquete estadístico $\mathrm{R}$ y en MINITAB. La Tabla 2 presenta una descripción de la variable rentabilidad de los activos, entendida como el porcentaje de ganancia del capital invertido en una cosecha de cebolla, la cual generalmente dura cinco meses.

Tabla 2. Descripción de la Rentabilidad de los Activos en las unidades productivas del DRACH

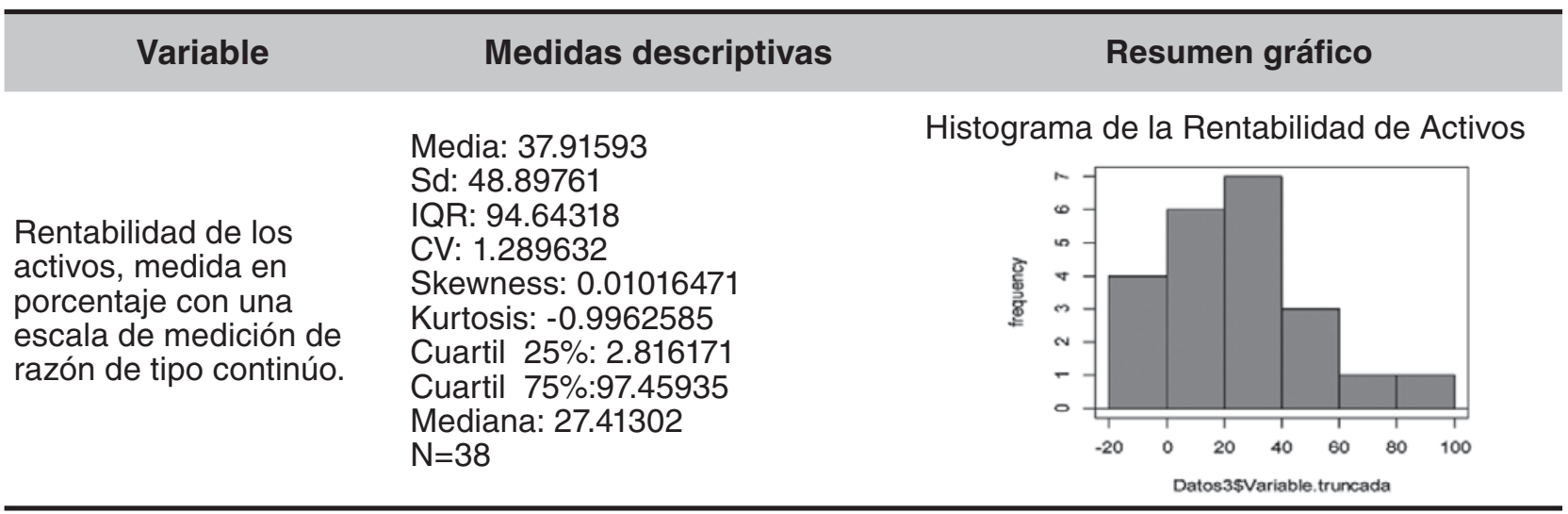

Los resultados indican que la rentabilidad media en las agroempresas estudiadas es de $37,92 \%$, con una desviación estándar de 48,90\%. Como se puede observar en el histograma y en las medidas numéricas se tiene una distribución de la rentabilidad simétrica con pico extremo y aislado, aspecto que influye en el alto coeficiente de variación: $128,96 \%$, indicando la existencia de una alta heterogeneidad respecto a la rentabilidad de los activos en las empresas seleccionadas.

Adicionalmente y tal como lo evidencian los dos picos de la distribución y el valor de la curtosis, -0.97, en el estudio se presentan varios tipos de empresas presentes respecto a la variable de estudio. Se distingue un primer grupo en el que se encuentran empresas cuyas rentabilidades han son negativas con valores entre 16 y $66 \%$, un segundo grupo con empresas con rentabilidades superiores al $100 \%$ y un tercer grupo en el que se localizan empresas con rentabilidades entre -50 y $15 \%$.
Teniendo en cuenta que la elección del estimador para construir el intervalo de confianza, que permitiera establecer los grupos de acuerdo a la metodología análisis de grupo y el test para determinar las diferencias significativas, dependía del modelo probabilístico supuesto, por tal razón se procedió a comprobar primero si la variable cumplía la condición de normalidad, utilizando la prueba de Shapiro Wilks por el tamaño de muestra pequeño. Se obtuvo un valor del estadístico $\mathrm{W}=0.938, \mathrm{p}$-value $=0.03605$, lo cual indicó con una significancia del $5 \%$, que la variable no sigue una distribución normal, por lo tanto se aplicaron en el análisis técnicas no paramétricas. Resulta apropiado describir la rentabilidad de activos en las agroempresas a través de la mediana, se encontró que el $50 \%$ de las empresas estudiadas tienen una rentabilidad inferior a $27.41 \%$. A partir del contraste de signos se determinó que la rentabilidad mediana está entre $12.13 \%$ y $50.91 \%$ con una confianza del $95 \%$. A partir de este intervalo se integraron los grupos cola, media y cabeza, los cuales se describen en la Tabla 3. 
Tabla 3. Descripción de la Rentabilidad de Activos por grupos en el DRACH

\begin{tabular}{|c|c|c|c|}
\hline Grupo & $\begin{array}{l}\text { Número y valores } \\
\text { de rentabilidad de } \\
\text { empresas del grupo }\end{array}$ & $\begin{array}{l}\text { Resumen numérico } \\
\text { de la rentabilidad }\end{array}$ & $\begin{array}{l}\text { Resumen gráfico de la } \\
\text { rentabilidad de activos }\end{array}$ \\
\hline Cabeza & $\begin{array}{l}100,00 ; 55,88 ; 100,00 \\
69,11 ; 100,00 ; 100,00 ; \\
100,00 ; 121,29 ; 100,00 \\
100,00 ; 100,00 ; 100,00 \\
89,84 \\
N=13\end{array}$ & $\begin{array}{l}\text { Mean 95,09; StDev 16,18; } \\
\text { CoefVar } 17,02 ; \text { Minimum 55,88 } \\
\text {; Maximum 121,29 } \\
\text { Grupo homogéneo caracteriza- } \\
\text { do por empresas con una ren- } \\
\text { tabilidad promedio de } 95,09 \% \text {. } \\
\text { Se tienen en este grupo tres } \\
\text { empresas atípicas las cuales } \\
\text { son } 55,88,69,11 \text { y } 121,29\end{array}$ & 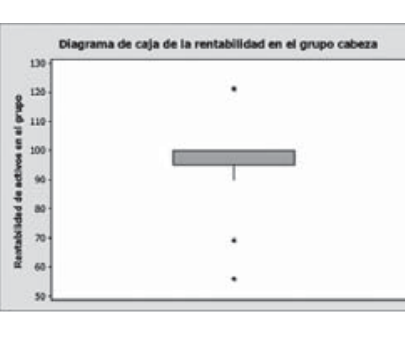 \\
\hline Media & $\begin{array}{l}16,17 ; 21,30 ; 25,37 ; \\
37,73 ; 36,61 ; 21,22 ; \\
29,45 ; 14,11 ; 55,88 ; \\
43,24 ; 39,30 ; 47,31 ; \\
15,25\end{array}$ & $\begin{array}{l}\text { Mean 31,00; StDev 13,41; } \\
\text { CoefVar 43,26; Minimum } \\
\text { 14,11; Maximum 55,88 } \\
\text { Grupo muy heterogéneo ca- } \\
\text { racterizado por empresas con } \\
\text { una rentabilidad promedio de } \\
31 \% \text {. }\end{array}$ & 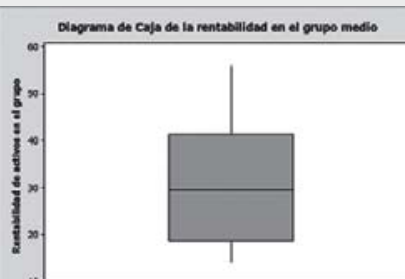 \\
\hline Cola & $\begin{array}{l}-52,11 ;-2,31 ;-10,09 ; \\
-22,11 ;-28,30 ; 4,74 ; 9,40 ; \\
-41,45 ; 2,17 ; \\
-10,68 ;-65,98 ;-14,71 ; \\
-15,66\end{array}$ & $\begin{array}{l}\text { Mean -19,01; StDev 22,69; } \\
\text { CoefVar 119,38; Minimum } \\
\text {-65,98; Maximum 9,40. } \\
\text { Grupo muy heterogéneo carac- } \\
\text { terizado por empresas con una } \\
\text { rentabilidad negativa promedio } \\
\text { de } 19,01 \% \text {. }\end{array}$ & $\mid$ \\
\hline
\end{tabular}

Con el objetivo de determinar si hay diferencias significativas al 95\%, en la rentabilidad mediana de los grupos propuestos, se aplicó el test no paramétrico de Kruskal Wallis, los resultados se presentan en la Tabla 4.

Tabla 4. Relación entre los grupos y la variable rentabilidad en el DRACH.

\begin{tabular}{llllll}
\hline & $\begin{array}{c}\text { Grupo } \\
\text { Cabeza }\end{array}$ & $\begin{array}{c}\text { Grupo } \\
\text { Media }\end{array}$ & $\begin{array}{c}\text { Grupo } \\
\text { Cola }\end{array}$ & Kruskal Wallis Chi-cuadrado & p- value \\
$\begin{array}{l}\text { Rentabilidad } \\
\text { de activos }\end{array}$ & 100.00 & 29.45 & -14.71 & Kruskal-Wallis chi-squared $=34.1181$, df $=2$ & $\begin{array}{l}\text { p-value }= \\
3.902 e-08\end{array}$ \\
\hline
\end{tabular}

Se observa que hay diferencias estadísticamente significativas $(p<<0,05)$, es decir, existen diferencias en las medianas de los grupos cabeza, media y cola.

Para tener resultados más confiables y aplicar la metodología análisis de grupo, se procedió a trabajar con una variable truncada al $15 \%$, organizar y tabular la información, y lo más importante identificar el factor clave RENTABILIDAD en cada una de las unidades productivas, la cual osciló entre $-20 \%$ y $89 \%$, en donde se obtuvieron 22 unidades productivas con el respectivo valor en el factor clave. 
Tabla 5. Valores de la rentabilidad en las unidades productoras de cebolla en el DRACH.

\begin{tabular}{lccccccccccc} 
Unidad & 2 & 3 & 4 & 6 & 8 & 9 & 10 & 12 & 13 & 14 & 15 \\
Rentabilidad & 16,67 & 21,30 & $-2,31$ & $-10,09$ & 25,37 & 37,73 & 36,61 & 4,74 & 21,22 & 9,40 & 29,45 \\
\hline & 16 & 17 & 18 & 19 & 21 & 22 & 23 & 24 & 29 & 31 & 38 \\
Unidad & 16 & & & & & & & & & & \\
Rentabilidad & 15,25 & 55,8 & 14,1 & 69,1 & 43,2 & 39,3 & 2,17 & $-10,68$ & 47,31 & $-14,71$ & 89,84 \\
\hline
\end{tabular}

En la Tabla 5, se puede observar, a través de la simbología de semáforo, las empresas ubicadas en los grupos tipo, de la metodología análisis de grupo, en donde se representan las empresas de la cola, con rentabilidades bajas y con producción de pérdidas; mientras que las empresas del grupo de la media por sus valores de rentabilidad fácilmente pueden llegar al grupo de la cabeza y las demás unidades pasan la prueba y están ubicadas en el grupo de la cabeza. A través del programa estadístico erre $(\mathrm{R})$ se corrieron los datos obtenidos en la rentabilidad de las unidades productivas de cebolla de bulbo, los resultados se presentan en la Tabla 6.

Tabla 6. Descripción de la Rentabilidad de los Activos/ variable truncada al $15 \%$ en las unidades productivas del DRACH

Variable

Medidas descriptivas

\section{Resumen gráfico}

Sd: 26,51

IQR: 32,999

CV: 1.07843

Skewness: 0,6050781

Kurtosis: 0.01655486

Intervalo de confianza de

la media al $95 \%$

activos, medida en porcentaje con una escala de medición de razón de tipo continúo. data: Datos\$ Variable. truncada

$\mathrm{t}=4.3493, \quad \mathrm{df}=21$

95 percent confidence interval:

LI: 12.83032

LS: 36.34238

Media $=24.58635$
Histograma de la Rentabilidad de Activos

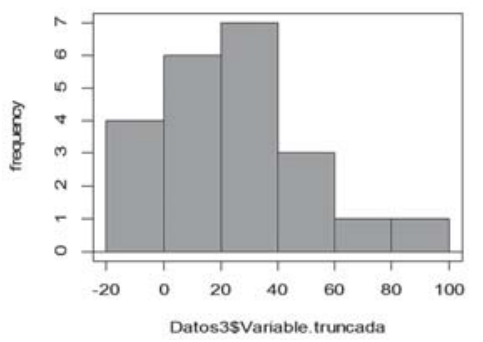

Según la metodología Análisis de Grupo, el primer paso consistió en aplicar la Prueba de Normalidad, la cual arrojó los siguientes resultados: Shapiro-Wilk normality test; data: Datos22\$Variable. truncada $\mathrm{W}=0.9651, \mathrm{p}$-valué $=0.5977$, Se puede inferir que la variable rentabilidad se distribuye según el modelo de probabilidad normal. Los resultados muestran, que los valores de la rentabilidad, van desde $-14,81 \%$ hasta $89,84 \%$; con una rentabilidad promedio en el agronegocio de la cebolla de bulbo del $24,58 \%$.
Luego de la prueba de normalidad y de establecer la descripción estadística, en los datos de análisis, se procedió a establecer el análisis de frecuencias y los intervalos de confianza para establecer los tres grupos tipo; para este caso, el intervalo del grupo de la media, está entre: Un límite inferior de LI: 12,83 y el límite superior LS: 36,34 ; lo que significa que las unidades productivas de cebolla que se encuentren en este rango, hacen parte del grupo de la media, y en la investigación corresponden a 6 unidades productivas; fue así como se 
hallaron los otros dos grupos tipo; en el grupo de la cabeza, se encuentran las que obtuvieron rentabilidades por encima de 36,34\%; correspondiendo a 8 unidades y en el grupo de la cola se ubican las unidades que obtuvieron rentabilidades por debajo del $12,83 \%$, para este caso fueron 8 unidades.
Hallados los tres grupos tipo, que propone la metodología, se establecen las conclusiones y se dan las recomendaciones respectivas para que las unidades que se encuentran en la cola, pasen a la media o a la cabeza y las unidades que están en la media pasen a la cabeza, como se indica en la Tabla 7.

Tabla 7. Indicadores Técnicos y Financieros del Análisis de grupo de 22 unidades productivas de cebolla en el DRACH.

\begin{tabular}{lccc}
\hline \multicolumn{1}{c}{ INDICADORES } & CABEZA 8 & MEDIA 6 & COLA 8 \\
1. TÉCNICOS & & & \\
\hline Área (ha) & 2,37 & 5,83 & 3,67 \\
& & & \\
Libras sembradas & 4,68 & 4,83 & \\
& & & 2 \\
No. cosechas año & 2 & 2 & \\
\hline 2. FINANCIERO & & & $-4,64 \%$ \\
\hline Rentabilidad \% & $52,37 \%$ & $21,35 \%$ & $-3,52 \%$ \\
Rentabilidad operativa. & $201,17 \%$ & $28,04 \%$ & $-3,52$ \\
Productividad Activo total. & 53,07 & 21,62 & $-30,05 \%$ \\
Productividad Planta y equipo. & 201,17 & $42,31 \%$ & $-30,56 \%$ \\
Margen Bruto. & $76,54 \%$ & $40,49 \%$ & $-33,24 \%$ \\
Margen Operativo & $76,47 \%$ & $40,29 \%$ & $\$ 57.600 .000$ \\
Margen Neto. & $75,28 \%$ & $\$ 104.400 .000$ & $\$ 23.040 .000$ \\
Costos de producción & $\$ 45.200 .000$ & $\$ 1.760 .000$ & $\$ 57.207 .500$ \\
Costo Mano de obra. & $\$ 17.705 .000$ & $\$ 169.333 .333,3$ & \\
Ingresos & $\$ 142.800 .000$ & & \\
\hline
\end{tabular}

\section{Análisis de los indicadores técnicos y financieros}

La producción de cebolla de bulbo en el departamento de Boyacá según el estudio realizado en 22 unidades estudiadas en el DRACH es una actividad rentable, se estableció una rentabilidad promedio de $24,58 \%$, teniendo en cuenta los ingresos y los costos explícitos e implícitos del cultivo.

A las unidades de producción de cebolla de bulbo tipificadas como grupos cola, media y cabeza se les realizó un análisis sintetizándose como sigue: Cola presentan características de tecnología tradicional y rudimentaria, utilizan mano de obra familiar no calificada, producen con recursos propios, con un área de minifundio $<1$ ha, con una capacidad de gestión y negociación precaria; situación que genera una rentabilidad baja $(-4,64 \%)$, con respecto a los otros dos grupos media y cabeza.

La rentabilidad de las unidades de producción del conjunto perteneciente a la Media es de 21,35\%, mientras que las del grupo de la Cabeza su rentabilidad es de 52,37\%; en razón al rubro costos de producción, el grupo de la Media, con costos promedio de $\$ 104.400 .000$ y Cabeza, con costos de producción promedio de $\$ 45.200 .000$ lo cual permite disminuir costos por compras en mayor escala y el extensivo manejo de capital en sus agronegocios. Para el resultado de las rentabilidades, 
se observó que se manejaban relaciones de producción (contrato de riesgo compartido, aparcería y explotación); tecnología más avanzada, siembras planificadas, recuperación de suelos e intensivo uso de agroquímicos y mano de obra contratada, con asistencia técnica profesional.

No obstante, que algunas unidades productivas muestran una baja rentabilidad, para los productores son rentables, pues ellos no tienen en cuenta los costos implícitos, en los que se encuentran las remuneraciones de los medios de producción que el agricultor no paga en realidad, pero en los que si se incurre; estos están relacionados con el trabajo familiar, la remuneración del propietario por su trabajo de administración, la renta de la tierra, los costos de depreciación y el costo de capital.

En el renglón de explotación de la cebolla de bulbo se presenta una gran incidencia de variables incontrolables como el clima y la estructura del mercado que generan alta fluctuación de los precios y variables controlables tales como la estacionalidad de la producción, la incipiente gestión y tecnología; factores que inciden en la dispersión de la rentabilidad, productividad y márgenes de utilidad.

\section{Conclusiones y recomendaciones}

En el estudio de rentabilidad de las unidades productoras de cebolla en el DRACH, se identificaron tres grupos tipo. Cada uno con niveles productivos y rentabilidades particulares, lo cual se evidencia en los valores de los indicadores financieros.

Para el grupo de la Cabeza la rentabilidad promedio se situó en $52,37 \%$, el grupo Media alcanzó $21,35 \%$ y para el grupo de la Cola fue de $-4.64 \%$.

Se evidenció que los porcentajes de las rentabilidades obtenidas están asociados a las prácticas de manejo del cultivo, a los costos de producción y al uso técnico de insumos y agroquímicos. En síntesis el indicador de rentabilidad determina la utilidad obtenida por cada peso invertido en el cultivo.
La productividad del activo muestra que por cada peso invertido en las unidades productoras de cebolla de bulbo del grupo de la Cola se generan ingresos negativos $(-4,15)$, y por lo tanto el nivel del agronegocio en esta categoría es ineficiente, aumentándose la susceptibilidad a la ocurrencia de eventos externos como las condiciones climáticas. El grupo de la categoría Media crea beneficios de $\$ 21,62$ por peso invertido y el grupo de la Cabeza obtiene ingresos $\$ 53,07$ por peso invertido, lo que se hace evidente en la gestión de los agronegocios, obteniéndose economías de escala en los cultivos y mejorando la utilidad y aprovechamiento de los recursos disponibles.

El margen bruto de ventas para cada uno de los grupos establecidos está dado así: para el grupo de la cabeza un valor del $76,47 \%$, para el grupo de la media de $46,47 \%$ y el grupo de la cola de $30,05 \%$; indicador que refleja que las áreas cultivadas tienen capacidad para convertir las ventas en utilidades.

Los costos de producción de la cebolla de bulbo se clasifican en costos explícitos (mano de obra, insumos, servicios y otros) y costos implícitos (trabajo familiar, arrendamiento, depreciación y renta del capital). La metodología implementada permitió el análisis de las unidades productivas del DRACH, desde el punto de vista de la gestión del agronegocio.

Se propone a los propietarios de las unidades productivas del grupo de la Cola, llevar a cabo una planificación de sus agronegocios implementando tecnología basada en la utilización y optimización del riego, uso apropiado de agroquímicos y realización de prácticas culturales oportunas

Para los grupos Media y Cabeza, se recomienda maximizar los recursos disponibles a la vez que se efectúe la introducción de tecnologías de punta p.ej. nutrición foliar para contrarrestar los efectos climatológicos adversos, creando mecanismos de defensa ante el ataque de entomopatogenos, de esta manera se aumentarán los indicadores de rentabilidad, productividad, márgenes de utilidad y el valor económico agregado EVA. 
Se recomienda adelantar programas de capacitación y motivación a los productores de cebolla de bulbo en los tres grupos de estudio Cabeza, Media y Cola, relacionados con la organización y la planeación de la actividad hortícola, con el propósito de disminuir la inestabilidad generada en los mercados y las altas tasas de variación en los precios que reciben el productor y consumidor. Igualmente, racionalizar la producción, implementando

\section{Literatura citada}

1. Alizaga, R. (2007). Apuntes de Contabilidad Agropecuaria, Ciudad de la Habana, Cuba. Recuperado de: http://www.contabilidad-agropecuaria/contabilidadagropecuaria.pdf

2. Álvarez, J. (2011).Uso de fertilizantes químicos y orgánicos en cebolla (Allium cepa $L$ ) en Apatzingán. Universidad Autónoma de México. México Distrito Federal.

3. Brian, F. (2010). Gerencia Financiera Efectiva. Editorial Kogan Page Ltda. Madrid, España.

4. Chombart, J. (2000). Metodología de análisis de grupos. Editorial Universo. Barcelona, España.

5. Corporación Autónoma Regional del Tolima (CORTOLIMA). (2006). Estudio de Factibilidad de Hortalizas. Ibagué, Tolima. Recuperado de: http://www.cortolima. gov.co/sites/default/files/images/stories/centro_documentos/recio/prospectiva_zonificacion_ambiental.pdf

6. Cristiano, S. \& Sastre, M. (1997). Radio inhibición de cebolla de bulbo: una estimación del beneficio económico para la zona de corto-rio Colorado. Universidad del Sur de Argentina. U.N.S. Tesis de grado. Provincia de Buenos Aires, Argentina.

7. Fonseca, J., Fonseca, A. \& Cleves, J. (2008). Manual técnico del cultivo de la cebolla puerro (Allium porrum L.) bajo el uso de buenas prácticas agrícolas (BPA) en el Distrito de Riego del Alto Chicamocha. DRACH. Duitama. Editorial Orión Lada. Bogotá, Colombia.

8. Fonseca, J. \& Cleves, J. (2010). Los sistemas de gestión de calidad: elemento para la competitividad en la producción de ajo y cebolla en Colombia. Simposio Internacional Del Ajo y Cebolla. Paipa. Editorial Produmedios.

9. García, O. (2009). Administración Financiera. Fundamentos y aplicaciones. IV Edición. Edit. Prensa Moderna Impresores S.A. Cali.

10. Lerdón, J. (2010). Contabilidad de Gestión Agropecuaria. Universidad Austral de Chile. Facultad de Ciencias Agrarias, Instituto de Economía Agraria. Valdivia, Chile.

11. Martínez, J. \& Alemán, V. (2004). El Enfoque de Sistemas en la Actividad Agropecuaria. Editorial Grupo Planeta México, Distrito Federal. México.

12. Martínez, E. (2009). Estudio de factibilidad para el establecimiento de un "packinghouse" para cebolla de bulbo (Allium cepa L.) en el Distrito de Riego del Alto Chicamocha. UNAL-UPTC. Bogotá. las buenas prácticas agrícolas, administrativas y la tecnología acorde con los requerimientos de las normas Global Gap que permitan mejorar la productividad y competitividad del producto considerando la articulación a los mercados internacionales, por lo tanto la asociatividad es un mecanismo transversal a las tres categorías con el propósito de manejar el mercadeo y obtener reducción en los precios de los insumos comprados.

13. Melo, L. (2008). Competitividad del sistema agroalimentario de la cebolla de bulbo con enfoque de cadena productiva en el Distrito de Riego del Alto Chicamocha (Boyacá). Tesis de grado, Convenio UNAL-UPTC. Tunja, Colombia.

14. Mena, C. (2012). Modelo de Gestión Financiera para la maximización de la empresa. Editorial Sangolqui. Madrid, España.

15. Ministerio de Agricultura y Desarrollo Rural (MADR). (2002). Programa Nacional de Transferencia de Tecnología Agropecuaria Pronatta. U.T. Bogotá, Colombia.

16. Ministerio De Agricultura y Desarrollo Rural. (MADR). 2009. Agronet. Evaluaciones Agropecuarias. Bogotá.

17. Ortiz, H. (2005). Gerencia financiera y diagnostico estratégico. Universidad Externado de Colombia. Bogotá.

18. Ortiz, H. (2006). Análisis financiero aplicado. Universidad Externado de Colombia. Bogotá.

19. PLAN HORTÍCOLA NACIONAL (PHN) - ASOHOFRUCOL. (2006). Canasta de productos del plan hortícola nacional. Cebolla de bulbo (Allium cepa L.). Corporación Colombia Internacional C.C.I. Bogotá, Colombia.

20. Pool, L. (2013). Propuesta metodológica para evaluar proyectos productivos con criterios locales de sustentabilidad en Calakmul, México. En Revista Avances de Investigación Agropecuaria, Vol. 17, N. 1 Universidad de Colima. México.

21. Puentes, G. \& Cleves, J. (2010). Análisis Financiero del Sistema de Producción de cebolla bulbo (Allium cepa L.) en el municipio de Corrales (Boyacá). Simposio internacional del ajo y la cebolla. Paipa. Editorial Produmedios.

22. Sociedad Colombiana de Ciencias Hortícolas (SCCH). (2009). Libro de resúmenes. Edit. Ruben's Impresores Editores. Bogotá

23. Ugalde, G. \& Esquivel, G. (1986). Administración de Empresas Agropecuarias. Revista Apuntes de Contabilidad Agropecuaria, Vol. 25 N. 1. Buenos Aires, Argentina.

24. Universidad de San Carlos. (2002). Rentabilidad de la producción del cultivo de la cebolla de bulbo. Editorial Rukemik Na'ojil. Ciudad de Guatemala. Guatemala. 